\title{
O USO DE SÍliCA GEL ORGANOFUNCIONALIZADA COMO AGENTE SEQUESTRANTE PARA METAIS
}

\section{Claudio Airoldi*}

Instituto de Química - Universidade Estadual de Campinas - CP 6154 - 13083-970 - Campinas - SP

Robson Fernandes de Farias

Departamento de Química - Universidade Federal de Roraima - UFRR - 69310-270 - Boa Vista - RR

Recebido em 19/8/99; aceito em 21/12/99

\begin{abstract}
THE USE OF ORGANOFUNTIONALIZED SILICA GEL AS SEQUESTRATING AGENT FOR METALS. An overview about the homogeneous and heterogeneous methods of synthesizing silylating agents and applications of the organofunctionalized silica-gel samples was explored. The pendant molecules attached covalently to the inorganic surface displayed important properties to act as sequestrating agents for metals in aqueous and non-aqueous solutions. The large variety of basic centres anchored on organic molecules improve the capacity in adsorbing cations. The increase in adsorption is dependent on the number of basic atoms disposed on the pendant molecules on the surface. The combination of acidic and basicity properties favours the selectivity, such is exemplified by the thiol modified silicas towards mercury (II) cations.
\end{abstract}

Keywords: silica; immobilization; cation; sequestrating agent.

\section{INTRODUÇÃO}

A sílica gel é um típico exemplo de polímero inorgânico que apresenta em sua composição grupos siloxanos, $\mathrm{Si}-\mathrm{O}-\mathrm{Si}$, em seu interior e tem vasta população de grupos silanóis, $\mathrm{Si}$ $\mathrm{OH}$, cobrindo toda a sua superfície ${ }^{1}$. A desigual distribuição de densidade eletrônica nesses últimos grupos, faz com que os mesmos manifestem um comportamento ácido, que é conhecido como ácido de Brфnsted. Desta forma, os sítios ácidos são os responsáveis pelo controle da reatividade que ocorre na superfície da sílica. Estes centros ácidos se distribuem aleatoriamente na superfície e dependendo da maneira como os grupos silanóis encontram-se dispostos, são denominados como vicinais, isolados ou geminais ${ }^{1}$. Uma representação simples destes três tipos de silanóis pode ser vista na Figura 1. Várias são as formas estruturais sob as quais este polímero inorgânico pode ser isolado. Porém, a estishovita ${ }^{2-4}$ é a única forma polimórfica não patogênica.

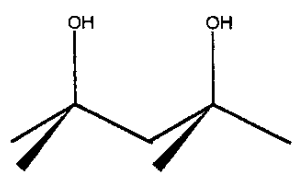

Silanóis vicinais

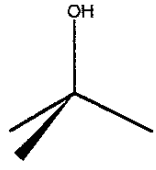

Silanol isolado

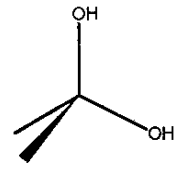

Silanóis geminais
Figura 1. Esquema dos tipos de grupos silanóis normalmente encontrados na superfície da sílica gel. O átomo central é o silício, ligados aos grupos silanóis.

A presença dos grupos silanóis na superfície da sílica ${ }^{5}$ foi detectatada pela primeira vez em 1936. Desde então, muitos estudos foram desenvolvidos e de maneira crescente, com o intuito de se calcular o número destes grupos, representado por $\alpha_{\mathrm{OH}}$, que normalmente é expresso por unidade de área superficial $\mathrm{em} \mathrm{nm}^{2}$. Para tanto, várias foram as técnicas empregadas

*e-mail airoldi@iqm.unicamp.br para tal determinação, porém, o valor de 5,0 OH/nm² é considerado como uma constante físico-química da sílica ${ }^{6}$, cujo valor também foi confirmado bem recentemente por termogravimetria ${ }^{7}$. Uma representação esquemática da distribuição aleatória de grupos silanóis na superfície unitária pode ser vista na Figura 2.

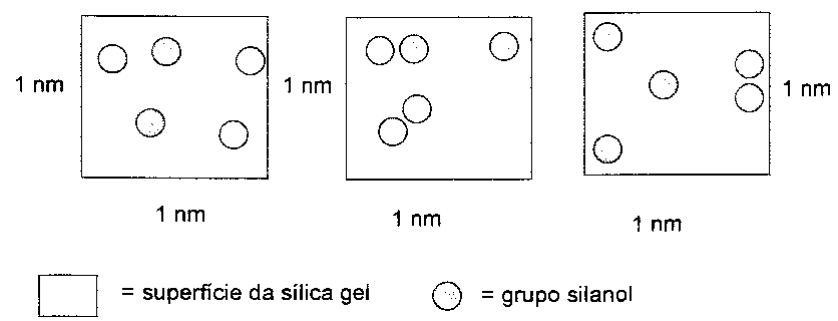

Figura 2. Distribuição de grupos silanóis na superfície de $1,0 \mathrm{~nm}^{2}$ da sílica.

Como os sítios ácidos de $B r \phi n s t e d^{8}$ respondem pela reatividade da sílica, é desejável que os silanóis estejam livres de possíveis interações antes de se efetuar quaisquer reações. Neste sentido, uma operação importante consiste na ativação da superfície da sílica mediante aquecimento, a fim de retirar as moléculas de água fisissorvidas. Porém, cuidados devem ser tomados porque, temperaturas acima de $200^{\circ} \mathrm{C}$, acarretam a condensação dos grupos silanóis, produzindo água, com consequente desidratação da sílica ${ }^{9-13}$, tornando-a assim pouco reativa. O processo de desidratação da superfície da sílica, resultando na formação de grupo siloxano é representado na Figura 3.

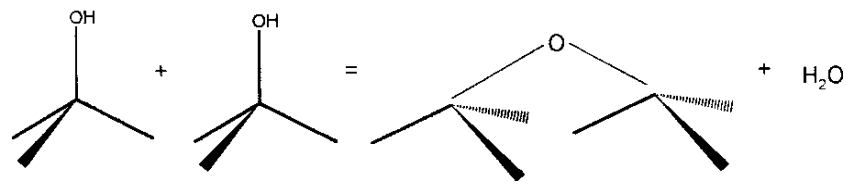

Figura 3. Desidratação da superfície da sílica, na formação do grupo siloxano. 
Uma maneira de se explorar a reatividade dos grupos silanóis na superfície consiste na reação de sililação, onde um alcoxissilano passa a ser quimicamente ligado à superfície, cuja operação é denominada de organofuncionalização ${ }^{14}$. Este tipo de reação pode ser genericamente representada como na Figura 4. Em verdade, por razões de natureza estérica, o átomo de silício do agente sililante normalmente encontra-se ligado a um ou no máximo a dois átomos de oxigênio do substrato inorgânico ${ }^{1}$.

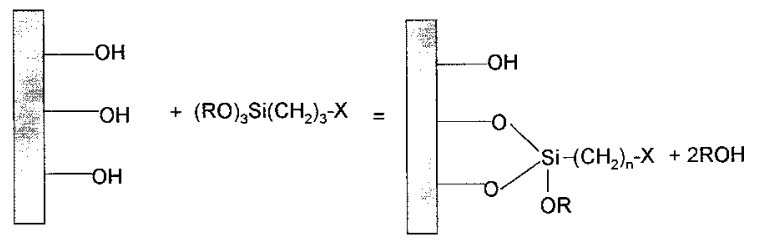

$\mathrm{R}=\mathrm{H}_{3} \mathrm{C}, \mathrm{H}_{3} \mathrm{C}-\mathrm{CH}_{2}$

$\mathrm{X}=\mathrm{Cl}, \mathrm{SH}, \mathrm{NH}_{2}, \mathrm{NCO}, \mathrm{NH}\left(\mathrm{CH}_{2}\right)_{2} \mathrm{NH}_{2}$, etc

Figura 4. Reação de sílica-gel com um agente sililante genérico $(\mathrm{OR})_{3} \mathrm{Si}\left(\mathrm{CH}_{2}\right)_{3}-\mathrm{X}$.

$\mathrm{O}$ agente sililante pode ser representado quimicamente por (RO) ${ }_{3} \mathrm{Si}\left(\mathrm{CH}_{2}\right)_{3} \mathrm{X}$, sendo $\mathrm{R}$ um radical alquila e $\mathrm{X}$ representa uma função orgânica do tipo $\mathrm{Cl}, \mathrm{SH}, \mathrm{NH}_{2}, \mathrm{NCO}, \mathrm{NH}\left(\mathrm{CH}_{2}\right)_{2} \mathrm{NH}_{2}$, etc. $\mathrm{Na}$ interação com o agente, a superfície passa a ser recoberta, com formação de ligação covalente $\mathrm{Si}-\mathrm{C}$, como mostra a Figura 4.

Do ponto de vista reacional os grupos silanóis livres, geminais ou vicinais podem interagir ${ }^{15,16}$ de maneira efetiva, além dos próprios grupos siloxanos ${ }^{17}$, dependendo das condições operacionais.

O desenvolvimento do campo de síntese de novos agentes sililantes impulsionou o processo de organofuncionalização da superfície de sílica gel, sendo envolvidas uma variedade de funções orgânicas inseridas nas cadeias e ancoradas covalentemente ao suporte. Desta forma, as propriedades destas sílicas modificadas quimicamente sofreram alterações, que puderam ser exploradas em várias atividades acadêmicas ou tecnológicas. Assim, a sílica organofuncionalizada encontra principalmente aplicações como fase estacionária para cromatografia $^{18-21}$ ou suporte para materiais com propriedades catalíticas $^{22-29}$. Uma destacada utilização consiste no uso da molécula imobilizada como agente sequestrante para metais, que será enfocado aqui.

A grande vantagem da utilização da sílica organofuncionalizada contendo o agente sequestrante advém da possibilidade de reciclagem do substrato adsorvente, além de apresentar propriedades relacionadas à grande estabilidade térmica, acessibilidade dos centros reativos, insolubilidade em meios orgânicos e ainda, não apresenta inchamento em solventes orgânicos.

Para se ter a molécula desejada sobre a superfície promovese primeiramente, a reação entre a sílica gel e o agente sililante e em seguida a reação desta superfície modificada com a espécie química, que contém o grupo coordenante, sendo este método chamado de heterogêneo. Em caso inverso, ocorre inicialmente a reação entre a espécie coordenante e o agente sililante, cuja nova molécula passa a ser ancorada na superfície do substrato inorgânico, caracterizando o método homogêneo ${ }^{11}$.

As moléculas mais comumente ancoradas apresentam oxigênio ou nitrogênio, ou mesmo ambos como centros de coordenação ${ }^{11}$, além de enxofre, fósforo, etc., sendo que, dependendo da rota de síntese, estas espécies químicas podem comportarem-se como mono ou polidentadas.

A adsorção de metais pelas sílicas modificadas pode ser efetuada em meio aquoso ou em solventes não aquosos, destacando-se principalmente o etanol e a acetona.

\section{ADSORÇÃO EM SÍLICA GEL}

De acordo com a IUPAC, a adsorção pode ser definida ${ }^{30}$ como o enriquecimento de um ou mais componentes numa camada interfacial, podendo ocorrer tanto a fisissorção como a quimissorção. Quando os efeitos atrativos que ocorrem entre o substrato e o adsorbato são provenientes de interações do tipo van der Waals, temos a fisissorção ${ }^{31}$, cuja energia de ligação substrato-adsorbato apresenta um valor entálpico médio de 20 $\mathrm{kJ} \mathrm{mol}^{-1}$. Já a quimissorção ${ }^{31}$ contrasta com o valor anterior, uma vez que as interações são de natureza iônica e/ou covalente entre o substrato e adsorbato, podendo chegar a um valor entálpico que varia de 250 a $500 \mathrm{~kJ} \mathrm{~mol}^{-1}$.

A quantidade de íons metálicos adsorvidos pode ser calculada utilizando-se a equação: $\mathrm{n}_{\mathrm{f}}=\left[\left(\mathrm{C}_{\mathrm{i}}-\mathrm{C}_{\mathrm{s}}\right) / \mathrm{m}\right] \mathrm{x} \mathrm{V}$, onde $\mathrm{n}_{\mathrm{f}}$ é o número de moles fixos na matriz, $\mathrm{C}_{\mathrm{i}}$ e $\mathrm{C}_{\mathrm{s}}$ são as concentrações inicial e sobrenadante dos íons metálicos, m é a massa de substrato e V é o volume de solução empregado no experimento. Um gráfico de $\mathrm{n}_{\mathrm{f}} / \mathrm{mmol} \mathrm{g}^{-1}$ em função de $\mathrm{C}_{\mathrm{s}} / \mathrm{mol} \mathrm{dm}^{-3}$ fornece um perfil típico de isoterma de adsorção, conforme ilustrado na Figura 5. A isoterma obtida invariavelmente segue um modelo como o apresentado, mostrando uma saturação dos grupos básicos das moléculas ancoradas, pelos centros ácidos metálicos. Para que se possa obter maiores informações a respeito do comportamento do sistema, normalmente é feito um ajuste dos dados à equação modificada de Langmuir: $\mathrm{C}_{\mathrm{s}} / \mathrm{n}_{\mathrm{f}}=\left(1 / \mathrm{n}^{\mathrm{s}} \mathrm{b}\right)+\left(\mathrm{C}_{\mathrm{s}}\right.$ $/ \mathrm{n}^{\mathrm{s}}$ ), a partir da qual pode-se obter a linearização da curva, que está também apresentada na mesma Figura 5. Nesta equação, $\mathrm{n}^{\mathrm{s}}$ é a capacidade máxima de adsorção e b é uma constante relacionada à constante de equilíbrio. Estes parâmetros são determinados através dos coeficientes angular e linear da reta obtida no

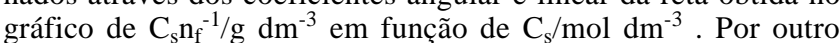
lado, o emprego dos valores de b também permite o cálculo da energia livre associada ao processo de adsorção ${ }^{32}$.

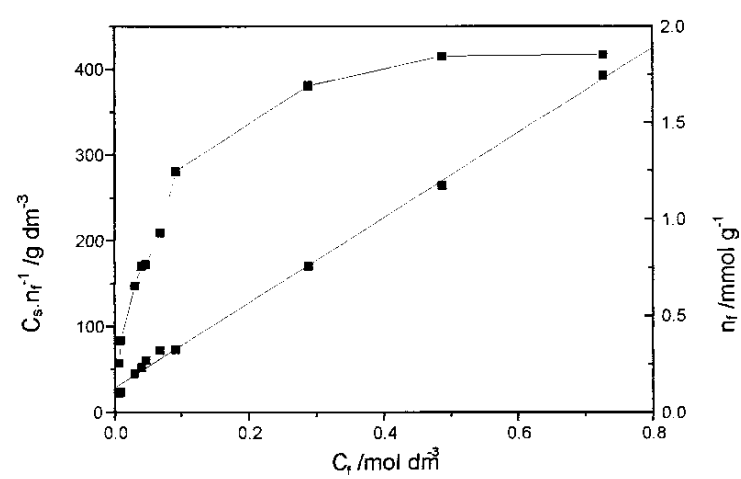

Figura 5. Representação de uma típica isoterma de adsorção $\left(n_{f}\right.$ em função de $C_{s}$ ), considerando-se sílica gel organofuncionalizada como substrato e ións metálicos como adsorbatos. Apresenta-se ainda a forma linearizada da curva $\left(C_{s} \cdot n_{f}^{-1}\right.$ em função de $\left.C_{s}\right)$, obtida pela aplicação da equação modificada de Langmuir.

Em função da presença dos sítios ácidos de Brönsted $^{33,34}$, mesmo não estando organofuncionalizada, a sílica gel mostrase capaz de adsorver as mais variadas espécies químicas ${ }^{35-41}$, como amidas $^{40}$, álcoois ${ }^{36}$, proteínas ${ }^{38}$ e fosfatos biologicamente $\operatorname{ativos}^{37}$.

Do ponto de vista químico, a sílica gel pode ser considerada como produto de síntese da condensação do ácido silícico, para dar a formação à uma estrutura amorfa. Esta mesma forma polimérica pode em determinadas condições, tornar-se cristalina, sendo que, na verdade, o método sintético parte de reagentes nas formas sódica ou potássica. Porém, quando acontece a troca destes cátions por prótons, dá-se a formação do chamado ácido silícico de fórmula $\mathrm{H}_{2} \mathrm{Si}_{20} \mathrm{O}_{42} \cdot \mathrm{xH}_{2} \mathrm{O}$. Um fato marcante deste ácido é a estrutura lamelar, que facilita a adsorção de álcoois ${ }^{42}$, 
alquilaminas ${ }^{43}$ e aminoácidos ${ }^{44}$, devido à facilidade de formação de ligações de hidrogênio entre os centros ácidos do polímero e os centros básicos das moléculas orgânicas polares. No caso da adsorção de álcoois, verifica-se a possibilidade de separar diferentes moléculas, em função de uma adsorção seletiva, através da adsorção na cavidade interlamelar ${ }^{42}$.

\section{O USO DO AGENTE SEQUESTRANTE IMOBILIZADO}

Os centros ácidos dos grupos silanóis da silíca gel são sensíveis à troca por íons metálicos. Um exemplo é o que acontece com o gadolínio e o ítrio trivalentes ${ }^{45}$, sendo que a força iônica da solução não afeta o perfil das isotermas de troca, porém, como é de se esperar, a extensão com que ocorre a troca depende do $\mathrm{pH}$ do meio reacional.

No processo de organofuncionalização da sílica gel podem ser incorporadas à sua superfície as mais variadas espécies, podendo ser simples ou complexas. No primeiro caso, tem-se os agentes sililantes encontrados comercialmente, que alocam na superfície funções químicas como haleto, cianeto, amina, etc. Já no segundo caso, são imobilizados polímeros condutores, tais como polianilina ${ }^{46}$ e fulerenos ${ }^{47}$. Este último é imobilizado mediante a prévia funcionalização da sílica com dietoximetilsilano. Com o auxílio de um precursor organossilano, torna-se possível também a imobilização de complexos ${ }^{48}$, como por exemplo, ${ }^{49}$ $(\mathrm{CO})_{2} \mathrm{Ni}\left[\mathrm{PPh}_{2}\left(\mathrm{C}_{6} \mathrm{H}_{4}\right) \mathrm{SiMe}_{2} \mathrm{Oet}\right]_{2}$.

Para explorar a capacidade de atuação do agente sililante na direta interação com cátions, os primeiros estudos mostram a organofuncionalização da sílica com alcoxissilanos, tal qual foram sintetizados, nos quais o grupo $X$ pode ser, por exemplo, amino, tiol, etc, conforme apresentado na Figura 4. Para efeito de ilustração, a Figura 6 contém uma representação esquemática da configuração da molécula de aminopropiltrimetoxissilano, após reação com a superfície da sílica. Como se nota, a flexibilidade da cadeia do agente imobilizado permite a interação do centro básico $\mathrm{NH}_{2}$ com o silanol do polímero inorgânico ${ }^{11}$.

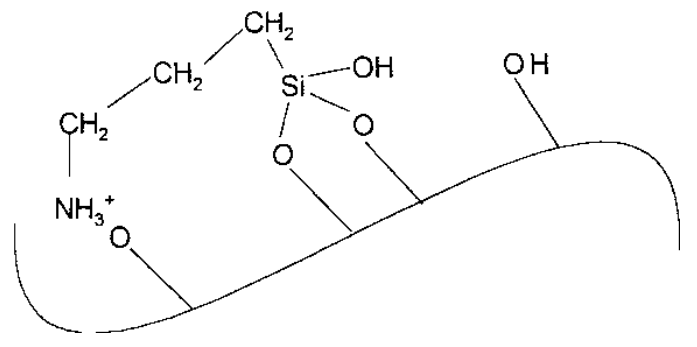

Figura 6. Esquema da conformação da molécula de aminopropiltrietoxissilano após imobilizada na superfície de sílica gel.

A presença de centros nucleofílicos nos agentes sililantes confere à superfícíe da sílica organofuncionalizada a capacidade de sequestrar metais. Por outro lado, agentes sililantes precursores como o cloropropil, $-\left(\mathrm{CH}_{2}\right)_{3}-\mathrm{Cl}$, são utilizados apenas, com a finalidade de permitir a posterior reação entre a sílica organofuncionalizada e moléculas que apresentem centros nucleofílicos, como por exemplo, aminas ${ }^{50}$.

Antes de se efetivar quaisquer processos com uma superfície ancorada, tem importância fundamental a perfeita caracterização do material em uso. Um procedimento largamente usado é a obtenção de espectros na região do infravermelho. Devido à própria absorção da matriz, fica apenas disponível uma janela entre 3500 a $1800 \mathrm{~cm}^{-1}$, o que fatalmente mascara as possíveis informações sobre grande parte das moléculas imobilizadas. A análise elementar deve ser criteriosamente interpretada devido à presença de grupos alcóxidos do agente sililante, que não se ligam à matriz, sendo altamente confiável a análise

de nitrogênio, que em muitos casos é efetuada utilizando-se o método de Kjedhal. Nos últimos anos, porém, com o avanço da técnica de ressonância magnética no estado sólido de vários núcleos, constata-se que os espectros de ${ }^{13} \mathrm{C}$ e ${ }^{29} \mathrm{Si}$ proporcionam resultados bastante confiáveis, no que diz respeito às ligações formadas entre o sililante e a superfície. Assim, um grande número de moléculas ancoradas na superfície da sílica gel aumenta sua capacidade de adorver metais ${ }^{51-73}$.

A densidade populacional de grupos ligados à matriz pode ser determinada por métodos clássicos de análise química, como titulação volumétrica, análise elementar de carbono, hidrogênio e nitrogênio, ou ainda por termogravimetria ${ }^{74}$. O grau de funcionalização geralmente atinge um valor médio de 1,0 mmol de grupos orgânicos ancorados por grama de sílica, sendo que, quanto maior o grau de recobrimento do substrato, maior o decréscimo do valor da área superficial ${ }^{50,63,64}$.

Três tipos de sílica gel com diferentes distribuições de poros foram utlizados na reação com o composto triclorossilino, considerado molécula como modelo. O efeito na ancoragem foi seguido através da técnica de espectroscopia na região do infravermelho com transformada de Fourrier com detecção foto-acústica ${ }^{15}$. Os dados obtidos demonstram que a reatividdae da sílica gel frente ao agente sililante é grandemente influenciada pelo diâmetro dos poros ${ }^{15}$. Nesta reação o triclorossilano reage exclusivamente com os grupos silanóis livres, quando o suporte foi previamente submetido à temperaturas elevadas, para ativação da superfície. A reatividade destes grupos livres é independente do diâmetro dos poros. Por outro lado, quando fazem ligações de hidrogênio passam a ser menos reativos à medida que o diâmetro dos poros diminui ${ }^{15}$.

Dentre os agentes sililantes mais simples, o 3-mercaptopropiltrimetoxissilano tem sido um dos mais utilizados na adsorção de cátions ${ }^{52,61,72,73}$. Porém, as sílicas ancoradas com o cloropropil e o aminopropil são mais amplamente utilizadas pelas facilidades em ampliar não só a cadeia orgânica, como também em aumentar o número de centros básicos na mesma, através de reações como as que estão representadas na Figura 7 .

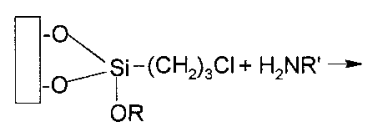

(a)

$\mathrm{R}=-\mathrm{CH}_{3},-\mathrm{CH}_{2} \mathrm{CH}_{3}$

$\mathrm{H}_{2} \mathrm{NR}^{\prime}=\mathrm{NH}_{2}(\mathrm{CO}) \mathrm{NH}_{2}, \mathrm{NH}_{2}\left(\mathrm{CH}_{2}\right)_{2} \mathrm{NH}_{2}, \mathrm{NH}_{2}-\mathrm{C}_{5} \mathrm{H}_{5} \mathrm{~N}$
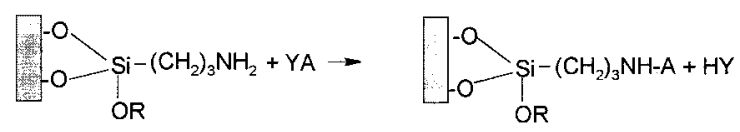

(b)
$\mathbf{R}=-\mathrm{CH}_{3},-\mathrm{CH}_{2} \mathrm{CH}_{3}$

$\mathrm{Y}=\mathrm{Br}, \mathrm{Cl}$

$\mathrm{A}=-\mathrm{Cl}(\mathrm{CO}) \mathrm{CH}_{3} \mathrm{l}_{2},-\mathrm{C}_{10} \mathrm{H}_{5} \mathrm{O}_{2} \mathrm{Cl},-\mathrm{C}_{9} \mathrm{H}_{9} \mathrm{O}_{3}$

Figura 7. Reações entre as sílicas modificadas com 3-cloropropiltrimetoxissilano (a) e 3-aminopropiltrimetóxissilano (b) com moléculas orgânicas. 
A sílica organofuncionalizada com o grupo mercaptopropil mostra-se capaz de adsorver $\mathrm{Ag}^{+}, \mathrm{Co}^{2+}, \mathrm{Co}^{3+}, \mathrm{Cu}^{2+}, \mathrm{Ni}^{2+}, \mathrm{Zn}^{2+}$, $\mathrm{Cd}^{2+}, \mathrm{Hg}^{2+}, \mathrm{Pb}^{2+}$ e $\mathrm{Fe}^{3+}$, sendo constatada a seletividade ${ }^{52,72}$ frente aos íons $\mathrm{Hg}^{2+}$. Em se utilizando amostras de sílica com diferentes porosidades e áreas superficiais, constata-se que a quantidade de íons $\mathrm{Hg}^{2+}$ adsorvidos aumenta até um máximo de $0,59 \mathrm{mmol} \mathrm{g}^{-1}$ de material ${ }^{72}$.

Mediante a reação prévia da sílica-gel com o agente sililante 3-cloropropiltrimetoxissilano consegue-se imobilizar uréia e seus derivados na superfície da sílica, utilizando-se o método de síntese tanto homogêneo como heterogêneo ${ }^{50,70}$. Verifica-se que a seletividade da matriz frente a um dado íon metálico depende do solvente utilizado ${ }^{50,70}$, considerando-se sempre um mesmo contra-íon para o cátion estudado. Diferentemente das sílicas modificadas com o grupo mercaptopropil $1^{52,72}$, a sílica modificada com uréia ou dimetiluréia apresenta uma maior capacidade de adsorção frente a cobre e níquel ${ }^{50}$ ou zinco e cobre ${ }^{70}$, do que mercúrio. Constata-se que a capacidade de adsorção relaciona-se, de um modo geral, com a energia livre de Gibbs calculada para o processo, mediante a utilização de dados calorimétricos ${ }^{50}$. Assim, pela utilização conjunta de sílicas modificadas com o grupo mercaptopropil e com uréia ou dimetiluréia, conseguir-se-ia separar, de forma bastante eficiente, uma mistura dos íons $\mathrm{Hg}^{2+}$ e $\mathrm{Cu}^{2+}$, por exemplo.

Pode-se ainda modificar a superfície da sílica promovendose a ancoragem de moléculas orgânicas tais como N-(2piridil)acetamida $^{62,71}$ e N-acil,N'-benzoiltiouréia ${ }^{67}$. Ambos tipos de sílicas modificadas apresentam maior capacidade de adsorção frente ao cobre, diferindo contudo, em relação ao zinco, cádmio e níquel.

Várias modificações da superfície da sílica foram feitas com ditiocarbamatos ${ }^{51,65}$ e em particular com propilpiperazinaditiocarbamato ${ }^{51}$, verificou-se a seguinte ordem de adsorção para íons trivalentes: $\mathrm{Cr}>\mathrm{Fe}>\mathrm{Co}$. Por sua vez, a matriz obtida pela reação entre a sílica modificada com N-(2aminoetil-3-aminopropil) trimetoxissilano e dissulfeto de carbono apresenta a seguinte capacidade de adsorção frente a íons divalentes: $\mathrm{Cu}>\mathrm{Zn}>\mathrm{Ni}>\mathrm{Co}$. A capacidade de adsorção diminui como consequência da relação metal:ligante para os complexos formados, sendo constatadas as razões 1:1 para cobre e zinco, já para níquel e cobalto esta relação passa a ser $1: 2$ e $1: 3$, respectivamente ${ }^{65}$.

$\mathrm{O}$ uso de aminas com diferentes números de centros básicos ancoarados na superfície da sílica ${ }^{60}$, sofrem influência na adsorção, tanto do solvente como dos contraânions dos cátions níquel, cobre e cobalto, quer na forma de cloreto, perclorato ou tetrafluoroborato, bem como na estrutura dos complexos formados. Estes fatores afetam a capacidade de adsorção dos compostos obtidos, cuja estabilidade dos complexos aumenta a medida que aumenta o número de centros básicos.

De um modo geral, as matrizes modificadas demonstram grande tendência em adsorver cobre e apresentam baixa afinidade por cobalto. Porém, a sílica-gel modificada com pentano2,4-diona ${ }^{66}$ contrasta com este tipo de comportamento, pois mostra a seguinte ordem de afinidade: $\mathrm{Zn}^{2+}>\mathrm{Cu}^{2+}=\mathrm{Co}^{2+}>$ $\mathrm{Ni}^{2+}$. Estes dados sugerem novamente a possibilidade de mediante a utilização conjunta de matrizes diferentemente modificadas, conseguir-se uma mistura de substratos altamente seletiva frente a íons metálicos específicos.

As sílicas organofuncionalizadas com acetilacetona ${ }^{69}$ e acetilidrazina $^{68}$ têm em comum o fato de apresentarem uma maior afinidade por cobalto (II) do que por zinco (II), quer em acetona ou em etanol.

A influência do ânion pode ser observada na adsorção de $\mathrm{Cu}^{2+}$ pela sílica-gel modificada com 2-amino-1,3,4-tiodiazol ${ }^{75}$, para a qual a adsorção de cobre (II) mostra-se na seguinte sequência para os contra-íons: cloreto $>$ brometo $>$ perclorato, quer em acetona ou em etanol, sendo maior no primeiro solvente.

$\mathrm{O}$ efeito interativo de vários cátions com moléculas contendo oxigênio, nitrogênio ou combinação de ambos centros básicos é menos pronunciado com enxofre, principalmente em meio não aquoso, são os exemplos mais típicos. Porém, é bastante limitado o número de estudos do ponto de vista energético e a Tabela 1 mostra apenas alguns dados para cobalto, cobre e mercúrio ${ }^{50,52,62,70}$. Para a reação do cátion, na forma $\mathrm{MX}_{2}$ com a superfície imobilizada em suspensão, Sup, em um determinado solvente, podemos escrever:

Sup $($ solvente $)+\mathrm{MX}_{2}($ solvente $)=\mathrm{SupMX}_{2}($ solvente $)+$ solvente

Este tipo de estudo requer primeiramente o conhecimento das isotermas obtidas através do sistema de batelada. Estes dados já ajustados ao modelo de adsorção de Langmuir são adaptados aos resultados das titulações calorimétricas. Os efeitos térmicos desta titulação são também ajustados ao mesmo modelo de adsorção, que possibilita à obtenção dos valores de adsorção máxima, variação de entalpia e os correspondentes valores das constantes de equilíbrio. Este último dado propicia o cálculo do valor de energia livre de Gibbs e consequentemente, a entropia do sistema. Os valores de $\Delta \mathrm{G}$ são normalmente exotérmicos e portanto, expressam o comportamento

Tabela 1. Entalpias, $\Delta \mathrm{H}$, capacidade de adsorção máxima, $\mathrm{N}^{\mathrm{s}}$, dos cátions cobalto, cobre e mercúrio (II) em etanol, acetona e água para algumas superfícies organofuncionalizadas, Sil-X $[X=$ uréia (u), metiluréia $(\mathrm{mu})$, dimetiluréia (dmu), N-(2-piridil)acetamida (piac), mercaptopropil (SH)].

\begin{tabular}{|c|c|c|c|c|c|}
\hline $\mathrm{MCl}_{2}$ & superfície & solvente & $\mathrm{N}^{\mathrm{s}} / 10^{-4} \mathrm{~mol} \mathrm{~g}^{-1}$ & $\Delta \mathrm{H} / \mathrm{kJ} \mathrm{mol}^{-1}$ & referência \\
\hline \multirow[t]{6}{*}{$\mathrm{Co}$} & Sil-dmu & etanol & 0,79 & $-89,60 \pm 0,07$ & 52 \\
\hline & & acetona & 1,16 & $70,79 \pm 0,11$ & 52 \\
\hline & Sil-u & etanol & 1,18 & $-7,12 \pm 0,01$ & 70 \\
\hline & & acetona & 2,43 & $25,51 \pm 0,08$ & 70 \\
\hline & Sil-mu & acetona & 1,13 & $46,64 \pm 0,10$ & 52 \\
\hline & Sil-piac & etanol & 1,62 & $-20,80 \pm 0,21$ & 62 \\
\hline \multirow[t]{6}{*}{$\mathrm{Cu}$} & Sil-dmu & etanol & 2,14 & $1,02 \pm 0,01$ & 52 \\
\hline & & acetona & 2,41 & $-4,43 \pm 0,01$ & 52 \\
\hline & Sil-u & etanol & 2,34 & $8,91 \pm 0,03$ & 70 \\
\hline & & acetona & 2,63 & $-28,38 \pm 0,04$ & 70 \\
\hline & Sil-SH & água & 0,81 & $-80,12 \pm 1,09$ & 50 \\
\hline & Sil-piac & etanol & 1,24 & $-127,98 \pm 1,28$ & 62 \\
\hline \multirow[t]{3}{*}{$\mathrm{Hg}$} & Sil-dmu & etanol & 1,61 & $-43,94 \pm 0,05$ & 52 \\
\hline & Sil-piac & etanol & 1,66 & $-80,66 \pm 0,81$ & 62 \\
\hline & Sil-u & etanol & 2,01 & $5,21 \pm 0,01$ & 70 \\
\hline
\end{tabular}


espontâneo das reações de adsorção. Os valores entálpicos associados aos processos de adsorção são normalmente mais favorecidos em solventes não aquosos, sendo quase sempre mais exotérmicos em etanol do que em acetona. Também, devido à desolvatação tanto dos cátions como da própria superfície ancorada, durante o processo interativo, os sistemas passam a ser favorecidos entropicamente.

O avanço dos aspectos sintéticos possibilitou a incorporação de uma variedade de moléculas no arcabouço da estrutura inorgânica polimérica da sílica, contendo diversos centros básicos em suas estruturas, no que resultou uma condição de uso destas superfícies como agentes quelantes ${ }^{76-114}$. Nestas matrizes, as espécies químicas responsáveis pela adsorção de metais apresentam nitrogênio $^{76-88}$, oxigênio ${ }^{89-99}$, ou enxofre ${ }^{100-114}$, como átomo doador.

Assim, amostras comerciais de etanol ${ }^{76}$, por exemplo, podem ser analisadas com relação aos íons $\mathrm{Cu}$, Ni e $\mathrm{Zn}$ (II) e Fe (III), utilizando-se sílicas modificadas com grupos 3(1imidazolil)propil obtendo-se resultados satisfatórios.

A adsorção de Pd(II) ${ }^{78,83,85,96,98,104,114}$ em sílicas organofuncionalizadas tem a capacidade de imobilizar quantidades que variam de 1,4 a $3,0 \times 10^{-4}$ mol de Pd(II) por grama de substrato. A representação esquemática de um complexo suportado ${ }^{114}$ é apresentada na Figura 8.

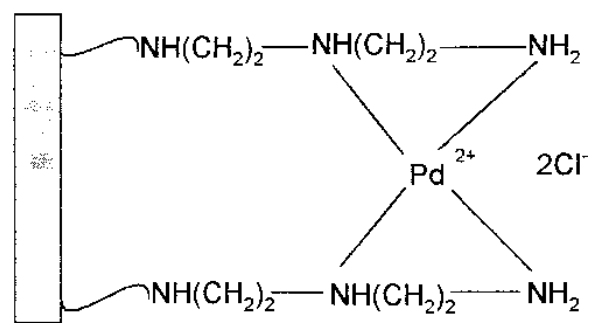

Figura 8. Complexo de Pd(II) suportado em sílica organofuncionalizada.

O emprego de sílicas organofuncionalizadas já contendo complexos de níquel, zinco ou cobre (II) suportados têm a capacidade de promover separações cromatográficas ${ }^{80,100,101,107,108}$, mostrado-se eficaz na resolução de separações de misturas de aminas aromáticas ${ }^{80}$, éteres e nitroalcanos ${ }^{101}$, por exemplo.

Uma nova fase estacionária foi preparada a partir de sílica imobilizada com etilenodiamina ${ }^{115}$. A mesma foi aplicada na separação de componentes de misturas sintéticas de aminas aromáticas e hidrocarbonetos polinucleares, através de cromatografia líquida de alta eficiência. Entretanto, a separação é afetada pela complexação dos centros básicos dos grupos pendentes pelo cobre (II). Quando a complexação ocorre, os grupos silanóis da sílica ficam mais expostos, resultando em uma fase mais polar, fato que é facilmente elucidado pelo espectro de ressonância magnética nuclear de silício 29. Na complexação as ligações de hidrogênio silanol-amina são rompidas, para formar ligações cobre-nitrogênio dos grupos pendentes. Consequentemente, a ordem de eluição dos compostos muda na presença da fase complexada com cobre, resultando em melhor separação das aminas aromáticas ${ }^{115}$.

Os cátions, ferro (III) $)^{82,86}$, chumbo (II) ${ }^{92}$, manganês (II) ${ }^{97}$, estrôncio e cálcio (II) ${ }^{88}$, platina (IV) ${ }^{88}$, lantânio e európio (III $)^{90}$, são íons também possíveis de serem separados mediante pré-concentração em sílicas organofuncionalizadas.

Um dos últimos avanços na utilização de sílicas organofuncionalizadas como agentes sequestrantes deve-se ao emprego de sílica lamelar ${ }^{116-120}$, obtida pela rota da diamina neutra ${ }^{121}$. Verifica-se através de dados calorimétricos e por difratometria de raios-x, que a capacidade de adsorção dessa matriz frente a cátions metálicos está diretamente relacionada às tranformações estruturais provocadas pelo processo de adsorção ${ }^{120,122}$. A seguinte ordem de capacidade de adsorção pode ser estabelecida para sílica a lamelar obtida utilizandose 1,12-diaminododecano como molécula modeladora: $\mathrm{Ni}^{2+}>$ $\mathrm{Cu}^{2+} \gg \mathrm{Co}^{2+}$. Representações esquemáticas da sílica lamelar, obtida pela rota da diamina neutra utilizando-se 1,12diaminododecano como molécula modeladora antes e após à coordenação com cobre ${ }^{2+}$, são apresentadas na Figura 9.

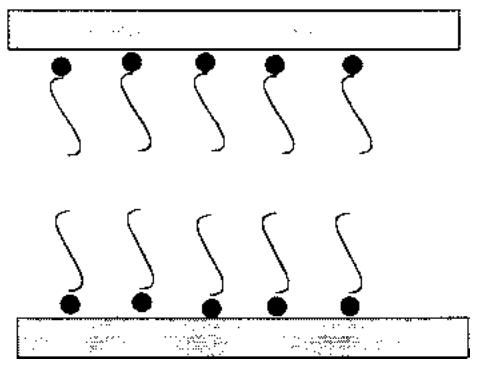

(a)

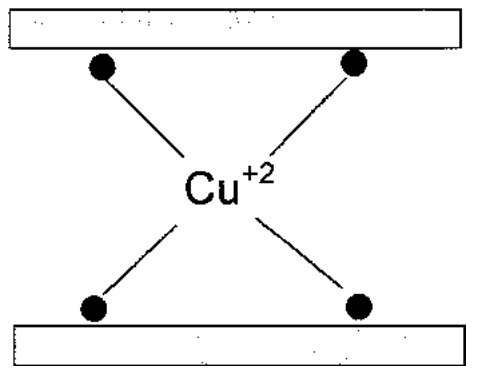

(b)

Figura 9. Sílica lamelar obtida pela rota da diamina neutra utilizando-se 1,21-diaminododecano como molécula modeladora antes (a) e após (b) coordenação com cobre (II).

\section{ALGUNS ASPECTOS RELACIONADOS COM A ADSORÇÃO DE METAIS}

A ressonância paramagnética de elétrons foi utilizada no estudo de compostos de cobre coordenado por centros complexantes de ditiocarbamatos suportados em sílica gel ${ }^{105}$. Os aspectos estruturais desta investigação tratam da obtenção de adutos, quando o suporte é colocado em presença de bases, cujas moléculas possuem átomos doadores de enxofre, nitrogênio ou oxigênio, tais como piridina, dibutilamina, morfolina e piperidina. Através dos resultados verifica-se que a reatividade entre estas últimas moléculas e os complexos suportados, é dependente dos valores de $\mathrm{pKa}$, além da influência do tempo de reação que sofre a geometria do aduto, como acontece com bases com $\mathrm{pKa}>5$. Por outro lado, as bases que apresentam valores de pKa menores que zero não reagem com os complexos suportados.

A sílica ancorada com etilenodiamina foi também utilizada como adsorvente, numa tentativa de correlacionar as propriedades de uma série de adsorventes com as espécies adsorvidas, bem como o pH da solução em que se processa a adsorção, e tendo como base a lei da ação das massas, chegou-se à equação ${ }^{83}$ :

$\log \beta-\mathrm{pK}=\log \alpha \cdot \mathrm{D}-\mathrm{pH}-\log \mathrm{C}_{\mathrm{L}}$

onde $\mathrm{K}$ é a constante de basicidade do grupo complexante, $\beta$ é a constante de estabilidade do complexo formado entre o íon adsorvido e o grupo adsorvente, o pH considerado é o da solução, D é o coeficiente de distribuição dos íons e $\mathrm{C}_{\mathrm{L}}$ é a concentração dos grupos complexantes no substrato. 
Assim, demonstra-se que o pH do meio, a concentração do cátion a ser sequestrado e o número de sítios de coordenação disponíveis no substrato, bem como a basicidade deste sítio coordenante, irão determinar a estabilidade termodinâmica do complexo formado, interferindo portanto, na seletividade da matriz.

A equação acima pode ser convenientemente utilizada para a estimativa das constantes de estabilidade dos complexos formados, em função do $\mathrm{pH}$, ou para selecionar as condições ótimas para a pré-concentração e separação de íons, desde que sejam conhecidas as constantes de estabilidade. Os dados obtidos mostram que as constantes de equilíbrio para os compostos formados, e portanto, as capacidades de adsorção, dependem do solvente utilizado ${ }^{62,70,75}$. Para a adsorção de $\mathrm{Cu}^{2+}$ em sílica modificada com 2-amino-1,3,4-tiadiazol ${ }^{75}$, por exemplo, verifica-se que as constantes de estabilidade dos complexos formados são maiores em acetona do que em etanol, exibindo a sequência: $\mathrm{Cl}^{-}>\mathrm{Br}^{-}>\mathrm{CIO}_{4}^{-}$.

\section{BREVE COMPARAÇÃO COM OUTROS SUPORTES ADSORVENTES}

Além da sílica gel organofuncionalizada, outros substratos, tais como os polímeros orgânicos quitina ${ }^{123}$ e quitosana ${ }^{124}$, zeólitos naturais ${ }^{125}$, certas argilas naturais como bentonita ${ }^{126}$, amostras de Latossolo roxo ${ }^{127}$ ou carvão ativado ${ }^{128}$ mostram-se capazes de adsorver metais. Neste processo a retirada de solução pode se dar mediante processo de complexação, tal como ocorre nas sílicas organofuncionalizadas, ou mediante processo de troca-iônica, em que o íon removido da solução é substituído por outro fornecido pelo substrato.

Os filossilicatos naturais ou sintéticos como magadeíta ${ }^{129}$, montmorrilonita ${ }^{130-133}$, caolinita ${ }^{134}$ e mica ${ }^{135}$ apresentam-se ainda como trocadores catiônicos e despertam grande interesse neste campo.

As isotermas de adsorção de cobre (II) obtidas com amostras de quitosana modificadas com glutaraldeído ${ }^{124}$, cuja adição deste reagente ao biopolímero aumenta a capacidade de adorção do produto final, mostram que o teor de adsorção difere daquele da quitosana de origem. Contudo, a quitosana sintética utilizada para modificação com glutaraldeído, exibe maior capaciddae de adsorção que as amostras modificadas. As capacidades de adsorção variam entre 0,10 e $0,45 \mathrm{mmol} \mathrm{g}^{-1}$, sendo portanto, comparável à capacidade exibida pela maioria das amostras de sílica modificadas.

$\mathrm{O}$ zeólito natural clinoptilolita ${ }^{125}$ exibe, frente à cobre e níquel divalentes, capacidades de troca de 0,56 e $0,25 \mathrm{meq} \mathrm{g}^{-1}$, respectivamente, sendo que as fases sódica e amoniacal apresentam capacidades de troca significativamente aumentadas, chegando-se a duplicar esta propriedade, em relação ao zeólito de partida.

Amostras de bentonita tanto natural como tratada quimicamente ${ }^{126}$ mostram-se eficientes na remoção de crômo (III) de águas residuais de curtumes. Verifica-se que a bentonita tratada com ácido sulfúrico $3,0 \mathrm{~mol} \mathrm{dm}^{-3}$ apresenta uma menor capacidade de troca do que a natural. A concentração de cátion chega a sofrer uma redução de 1850 para $25 \mathrm{ppm}$, dependendo das condições experimentais, com a água residual exibindo um

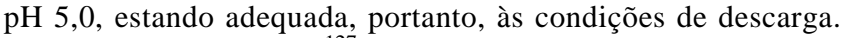

O Latossolo vermelho ${ }^{127}$, rico em ferro, oriundo principalmente da magnetita, caolinita e gibsita, mostra-se como um trocador iônico com os cátions divalentes de cobre, zinco, cádmio, mercúrio e cálcio, além do sódio. A magadeíta por sua vez ${ }^{129}$, mostrase como trocador frente ao par $\mathrm{H}^{+} / \mathrm{Na}^{+}$, com o processo de troca exercendo marcantes efeitos sobre a estrutura da matriz.

Um estudo recente ${ }^{135}$ demonstrou que a forma sódica da mica pode apresentar-se como exelente trocador iônico frente aos cátions divalentes $\mathrm{Cd}, \mathrm{Ni}, \mathrm{Co}, \mathrm{Mn}$ e $\mathrm{Zn}$, exibindo capacidade de troca da ordem de 2 a $3 \mathrm{meq}^{-1}$, dando a seguinte ordem se seletividade: $\mathrm{Zn}^{2+}>\mathrm{Ni}^{2+} \approx \mathrm{Co}^{2+} \approx \mathrm{Cd}^{2+}>\mathrm{Mn}^{2+}$.

\section{CONCLUSÃO}

O uso de sílica gel convenientemente explorada permite a sua organofuncionalização, que por sua vez pode ser eficazmente empregada como reagente sequestrante para metais em solução.

Verifica-se que, em função do grupo coordenante ancorado, a quantidade de íons metálicos adsorvidos por grama de amostra pode ser controlada, bem como a seletividade do processo de adsorção.

O controle da seletividade pode ser exemplificado em relação aos íons $\mathrm{Hg}^{2+}$, que exibem adsorção preferencial em sílicas modificadas com o grupo mercaptopropil ${ }^{49,71}$, em função da similaridade de dureza entre o ácido mole mercúrio e a base enxofre ${ }^{49}$. Também podem ser obtidas matrizes com maior afinidade por cobre e zinco $^{64}$. Assim, pelo uso combinado de diversas matrizes de sílicas organofuncionalizadas pode-se conseguir uma separação bastante eficiente entre diferentes cátions metálicos, ampliando assim as possibilidades de aplicação analítica destas interessantes matrizes inorgânicas quimicamente modificadas.

\section{AGRADECIMENTOS}

Os autores agradecem à CAPES-PICDT e ao CNPq pelas bolsas recebidas e à FAPESP pelo suporte financeiro.

\section{REFERÊNCIAS}

1. Iler, R. K., The Chemistry of Silica, John Wiley \& Sons, New York, 1979.

2. Cerrato, G.; Fubini, B.; Baricco, M.; Moterra,C.; J. Mater. Chem. 1995, 5, 1935.

3. Wiessner, J. H.; Mandel, N. S.; Sohnle, P. G.; Hasegawa, A.; Mandel, G. S.; Am. Rev. Resp. Dis. 1990, 141, 111.

4. Wiessner, J. H.; Henderson, J. D. Jr.; Sohnle, P. G.; Mandel, N. S.; Mandel, G. S.; Am. Rev. Resp. Dis. 1988, $138,445$.

5. Kiselev, A. V.; Kollodn. Zh. 1936, 2, 17.

6. Zhuravlev, L. T.; Langmuir 1987, 3, 316.

7. Farias, R. F. de, Airoldi, C.; J. Thermal Anal. 1998, 53, 751.

8. Gates, B. C., Catalytic Chemistry, John Wiley \& Sons, New York, 1992.

9. Van Der Voort, P.; Gillis-D'Hamers, I.; Vansant, E. F.; J. Chem. Soc., Faraday Trans. 1990, 86, 3751.

10. Lochmuller, C. H.; Kersey, M. T.; Langmuir 1988, 4, 572.

11. Leyden, D. E. (Editor), in Silanes, Surfaces and Interfaces, Gordon and Breach Science Publishers, New York, 1986.

12. Peri, J. B.; Hensley Jr.; A. L.; J. Phys. Chem. 1966, 70, 3941.

13. Carman, P. C.; Trans. Faraday Soc. 1940, 36, 964.

14. Arakaki, L. N. H.; Airoldi, C.; Quim. Nova 1999, 22, 246.

15. Van Der Voort, P.; Gillis-D'Hamers, I.; Vrancken, K. C.; Vansant, E. F.; J. Chem. Soc., Faraday Trans. 1991, 87, 3899.

16. Hair, M. L.; Hertl, W.; J. Phys. Chem. 1969, 73, 4269.

17. Gillis-D'Hamers, I.; Philippaerts, J.; Van Der Voort, P.; Vansant; E. F.; J. Chem. Soc., Faraday Trans. 1990, 86,3747

18. Björklund, M.; Hearn, M. T. W.; J. Chromatogr. A 1996, 728,149

19. Lin, C.-E.; Li, F.-K.; Lin, C.-H.; J. Chromatogr. A 1996, $722,211$.

20. Mifune, M.; Shimomura, Y.; Saito, Y.; Mori, Y.; Onoda, M.; Iwado, A.; Motohashi, N.; Haginaka, J.; Bull. Chem. Soc. Jpn. 1998, 71, 1825.

21. Oliveira, S. F.; Espinola, J. G. P.; Lemus, W. E. S.; Souza, A. G. de; Airoldi, C.; Colloids Surf. A 1998, 136, 151.

22. Butterworth, A. J.; Clark, J. H.; Walton, P. H.; Barlow, S. J.; Chem. Commun. 1996, 1859.

23. Harmer, M. A.; Sun, Q.; Michalczyk, M. J.; Yang, Z.; Chem. Commun. 1997, 1803. 
24. Collman, J. P.; Belmont, J. A.; Brauman, J. I.; J. Am. Chem. Soc. 1983, 105, 7288.

25. Clark, J. H.; Macquarrie, D. J.; Chem. Commun. 1998, 853.

26. Rao, Y. V. S.; De Vos, D. E.; Bein, T.; Jacobs, P. A.; Chem. Commun. 1997, 355.

27. Wilson, K.; Clark, J. H.; Chem. Commun. 1998, 2135.

28. Lim, M. H.; Blanford, C. F.; Stein, A.; Chem. Mater. 1998, 10, 467.

29. Chisem, I. C.; Rafelt, J.; Shieh, M. T.; Chisem, J.; Clark, J. H.; Jachuck, R.; Macquarrie, D.; Ramshaw, C.; Scott, K.; Chem. Commun. 1998, 1949.

30. Sing, K. S. W.; Everet, D. H.; Haul, R. A. W.; Moscou, L.; Pierotti, R. A.; Rouquérol, J.; Siemieniewska, T.; Pure Appl. Chem. 1985, 57, 603.

31. McQuarrie, D. A.; Simon, J. D.; Physical Chemistry, a molecular approach, University Science Books, Sausalito, 1997.

32. Nunes, L. M.; Airoldi, C.; Thermochim. Acta 1999, 328, 297.

33. Iamail, H. M.; Mansour, S. A. A.; Zaki, M. I.; Thermochim. Acta 1992, 202, 269.

34. Takei, T.; Eriguchi, E.; Fuji, M.; Watanabe, T.; Chikazawa, M.; Thermochim. Acta 1998, 308, 139.

35. Staszczuk, P.; Thermochim. Acta 1998, 308, 147.

36. Horr, T. J.; Ralston, J.; Smart, R. St. C.; Colloids Surf. A 1992, 63, 21.

37. Hamdani, K.; Cheng, K. L.; Colloids Surf. A 1992, 63, 29.

38. Ito, M.; Yamauchi, K.; Matsuzawa, K.; Colloids Surf. A 1993, 74, 107.

39. Goworek, J.; Nieradka, A.; J. Thermal Anal. 1996, 46, 417.

40. Airoldi, C.; Santos Jr., L. S.; Thermochim. Acta 1986, 104,111

41. Ahmad, I.; Dines, T. J.; Anderson, J. A.; Rochester, C. H.; J. Colloid Interface Sci. 1997, 195, 216.

42. Döring, J.; Lagaly, G.; Beneke, K.; Dékány, I.; Colloids Surf. A 1993, 71, 219.

43. Döring, J.; Lagaly, G.; Clay Minerals 1993, 28, 39.

44. Beneke, K.; Lagaly, G.; Amer. Miner. 1989, 74, 224.

45. Kosmulski, M.; J. Colloid Interface Sci. 1997, 195, 395.

46. Wu, C.-G.; Chen, J. Y.; Chem. Mater. 1997, 9, 399.

47. Planeix, J.-M.; Coq, B.; Menorva, L.-C.; Medina, P.; Chem. Commun. 1996, 2087.

48. Hwang, K.-O.; Sasaki, T.; J. Mater. Chem. 1998, 8, 2153.

49. Behringer, K. D.; Blümel, J.; Chem. Commun. 1996, 653.

50. Vieira, E. F. S.; Simoni, J. de A.; Airoldi, C.; J. Mater. Chem. 1997, 7, 2249.

51. Fonseca, M. G.; Espínola, J. G. P.; Oliveira, S. F.; Santos, L. C. R. dos; Souza, A. G. de, Airoldi, C.; Colloids Surf. A 1998, 133, 205.

52. Santos, M. R. M. C.; Airoldi, C.; J. Colloid Interface Sci. 1996, $183,416$.

53. Barradas, E. F. M.; Cestari, A. R.; Airoldi, C.; Buffon, R.; Braz. J. Chem. Eng. 1998, 15, 146.

54. Lim, M. H.; Blanford, C. F.; Stein, A.; Chem. Mater. 1998, 10, 467.

55. Alcântara, E. F. C.; Airoldi, C.; Nakamura, O.; Vargas, H.; Paixão, F.; Polyhedron 1992, 11, 1701.

56. Cestari, A. R.; Airoldi, C.; Langmuir 1997, 13, 2681.

57. Hoorn, H. J.; e Joode, P.; Dijkstra, D. J.; Driessen, W. L.; Kooijman, H.; Veldman, N.; Spek, A. L.; Reedijk, J.; J. Mater. Chem. 1997, 7, 1747.

58. Jaroniec, C. P.; Gilpin, R. K.; Jaroniec, M.; J. Phys. Chem. 1997, 101, 6861.

59. Taylor, I.; Howard, A. G.; Anal. Chim. Acta 1993, 271, 77.

60. Lishko, T. P.; Glushchenko, L. V.; Kholin, Y. V.; Zaitsev, Z. N.; Bugaevskii, A. A.; Donskaya, N. D.; Shevchenko, T. G.; Russ. J. Phys. Chem. 1991, 65, 2996.

61. Cestari, A. R.; Airoldi, C.; J. Braz. Chem. Soc. 1995, 6, 291.

62. Airoldi, C.; Alcântara, E. F. C.; J. Chem. Thermodyn. 1995, 27, 623 .

63. Airoldi, C.; Gonçalves, A. S.; Colloids Surf. 1987, 28, 199.
64. Gonçalves, A. S.; Airoldi, C.; Polyhedron, 1989, 8, 2901.

65. Espínola, J. G. P.; Freitas, J. M. P. de, Oliveira, S. F. de, Airoldi, C.; Colloids Surf. A 1994, 87, 33.

66. Espínola, J. G. P.; Freitas, J. M. P. de, Oliveira, S. F. de, Airoldi, C.; Colloids Surf. A 1992, 68, 261.

67. Costa, C. M. M.; Pesco, C.; Quim. Nova 1993, 16, 18.

68. Airoldi, C.; Alcântara, E. F. C.; Colloids Surf. A 1989, 39, 291.

69. Airoldi, C.; Alcântara, E. F. C.; Thermochim. Acta 1995, $259,95$.

70. Airoldi, C.; Santos, M. R. M. C.; J. Mater. Chem. 1994, 4, 1479.

71. Airoldi, C.; Alcântara, E. F. C.; Nakamura, O.; Paixão, F. J. da; J. Mater. Chem. 1993, 3, 479.

72. Brown, J.; mercier, L.; Pinnavaia, T. J.; Chem. Commun. 1999, 69 .

73. Cestari, A. R.; Airoldi, C.; J. Colloid Interface Sci. 1997, 195,338

74. Cestari, A. R.; Airoldi, C.; J. Thermal Anal. 1995, 44, 79.

75. Dias Filho, N. L.; Gushikem, Y.; Franco, D. W.; Schultz, M. S.; Vasconcelos, L. C. G.; Colloids Surf. 1998, 141, 181.

76. Moreira, J. C.; Gushikem, Y.; Anal. Chim. Acta 1985, 176, 263.

77. Skopenko, V. V.; Zaitsev, V. N.; Trofimchuk, A. K.; Soviet Progr.Chem. 1985, 51, 1.

78. Leyden, D. E.; Luttrell, G. H.; Anal. Chem. 1975, 47, 1612.

79. Köklü, Ü.; Chim. Acta Turcica 1984, 12, 265.

80. Pyell, U.; Stork, G.; Fres. J. Anal. Chem. 1992, 343, 576.

81. Romanovskii, B. V.; Kireev, S. G.; Russ. J. Phys. Chem. 1987, 61, 218.

82. Skopenko, V. V.; Trofimchuk, A. K.; Zaitsev, V. N.; Russ.; J. Inorg. Chem. 1982, 27, 1458.

83. Kudryavtsev, G. V.; Ivanov, V. M.; Lisichkin, G. V.; Doklay Chem. 1980, 250, 39.

84. Mil'chenko, D. V.; Kudryavtsev, G. V.; Ponomarev, D. V.; Lisichkin, G. V.; Russ. J. Phys. Chem. 1987, 61, 2823.

85. Suzuki, T.; Tsunoda, K.-I.; Akaiwa, H.; Chem. Lett. 1994, 881.

86. Kholin, Yu. V.; Zaitsev, V. N.; Donskaya, N. D.; Russ. J. Inorg. Chem. 1990, 35, 891.

87. Skopenko, V. V.; Zaitsev, V. N.; Kholin, Yu. V.; Bugaevslii, A. A.; Russ. J. Inorg. Chem. 1987, 32, 970.

88. Skopenko, V. V.; Lishko, T. P.; Sukhan, T. A.; Trofimchuk, A. K.; Soviet Prog. Chem. 1985, 51, 5.

89. Lisichkin, G. V.; Kudryavtsev, G. V.; Nesterenko, P. N.; J. Anal. Chem. USSR 1984, 38, 1288.

90. Voronina, R. D.; Kudryavtsev, G. V.; Lisichkin, G. V.; Runov, V. K.; Russ. J. Phys. Chem. 1985, 59, 294.

91. Hoorn, H. J.; de Joode, P.; Dijkstra, D. J.; Driessen, W. L.; Kooijman, H.; Veldman, N.; Spek, A. L.; Reedijk, J.; J. Mater. Chem. 1997, 7, 1747.

92. Terada, K.; Inoue, A.; Inamura, J.; Kiba, T.; Bull. Chem. Soc. Jpn. 1977, 50, 1060.

93. Pyell, U.; Stork, G.; Fresenius J. Anal. Chem. 1992, 342, 376.

94. Rykowska, I.; Wawrzyniak, R.; Wasiak, W.; Chem. Anal. 1994, 39, 335.

95. Nesterov, M. A.; Lafer, L. I.; Dykh, Zh. L.; Sharf, V. Z.; Yakerson, V. I.; Bull. Acad. Sci. USSR 1987, 36, 19.

96. Kudryavtsev, G. V.; Russ. J. Phys. Chem. 1987, 61, 468.

97. Zaitsev, V. N.; Trofimchuk, A. K.; Soviet Progr. Chem. 1984, $50,5$.

98. Wasiak, W.; Rykowska, I.; J. Chromatogr. A 1996, 723, 313.

99. Wasiak, W.; Rykowska, I.; J. Chromatogr. A 1997, 773, 209.

100. Woltermann, G. M.; Durante, V. A.; Inorg. Chem. 1983, 22,1954

101. Skopenko, V. V., T. P. Lishko, T. A.; Sukhan, A. K. Trofimchuk, Trachevskii, V. V.; Russ. J. Inorg. Chem. 1981, 26, 1331. 
102. Aydin, A.; Köklü, Ü, Tascioglu, S.; Chim. Acta Turcica 1987, 15, 91

103. Mahmoud, M. E.; Soliman, E. M.; Talanta 1997, 44, 1063.

104. Belyakova, L. A.; Lyashenko, D. Yu.; Russ. J. Gen. Chem. 1997, 67, 1321.

105. Solozhenkin, A. P. M.; Baratova, Z. R.; Lisichkina, O. A.; Semikopnyi, A. I.; Doklady Phys. Chem. 1991, $316,147$.

106. Akman, S.; Ince, H.; Köklü, Ü, Anal. Sci. 1991, 7, 799.

107. Den Bleyker, K. T.; Sweet, T. R.; Chromatographia 1980, 13,114

108. Cauzzi, D.; Predieri, G.; Tiripicchio, A.; Zanoni, R.; Giori, C.; Inorg. Chim. Acta 1994, 221, 183.

109. Cauzzi, D.; Marzolini, G.; Predieri, G.; Tiripicchio, A.; Costa, M.; Salviati, G.; Armigliato, A.; Basini, L.; Zanoni, R.; J. Mater. Chem. 1995, 5, 1375.

110. Wasiak, W.; Chromatographia 1995, 41, 107.

111. Capka, M.; Czakoová, M.; Hillerová, E.; Paetzold, E.; Oehme, G.; J. Mol. Cat. A 1995, 104, L123.

112. kholin, Yu. V.; Func. Mater. 1995, 2, 23.

113. Skopenko, V. V.; Kholin, Yu. V.; Zaitsev, V. N.; Mernyi, S. A.; Konyaev, D. S.; Russ.; J. Phys. Chem. 1993, 67, 658.

114. Zaitsev, V. N.; Kholin, Yu. V.; Konyaev, D. S.; Russ. J. Inorg. Chem. 1993, 38, 947.

115. Silva, C. R.; Jardim, I. C. S. F.; Airoldi, C.; HRC- J. High Res. Chromatogr. 1999, 22, 103.

116. Farias, R. F. de, Airoldi, C.; Quim. Nova 2000, 23, 88

117. Farias, R. F. de, Airoldi, C., IX Brazilian Meeting on Inorganic Chemistry, Angra dos Reis, 1998.

118. Farias, R. F. de, An. Assoc. Bras. Quim. 1999, 48, 112.
119. Farias, R. F. de, Airoldi, C.; J. Non-Cryst. Solids 2000 , 261,181

120. Farias, R. F. de, Airoldi, C.; J. Solid State Chem. 2000, 149, 113

121. Tanev, P. T.; Pinnavaia, T. J.; Science 1996, 271, 1267.

122. Farias, R. F. de; Airoldi, C.; Colloids Surf. A, no prelo.

123. Monteiro Jr., O. A. C.; Airoldi, C.; J. Colloid Inteface Sci. 1999, 212, 212.

124. Monteiro Jr., O. A. C.; Airoldi, C.; J. Biol. Macromol. 1999, 26, 119.

125. Estupinán, A.; Sarmiento, D.; Gavis, A. M. B. de, Rev. Colom. Quím 1998, 27, 31.

126. Isáza, A. E.; casafús, M. M.; Ochoa, C. R.; Martínez, C. T.; Molina, C. S.; Rev. Colom. Quím 1998, 27, 83.

127. Airoldi, C.; Critter, S. A. M.; Clays Clay Miner. 1997, 45,125

128. Reed, B. E.; Matsumoto, M. R.; Sep. Sci. Technol. 1993, $28,2179$.

129. Rojo, J. M.; Ruiz-Hitzky, E.; Sanz, J.; Inorg. Chem. 1988, $27,2785$.

130. Kijima, T.; Nakazawa, H.; Kobayashi, M.; Bull. Chem. Soc. Jpn. 1988, 61, 4277.

131. Lahav, N.; Lacher, M.; Yariv, S.; J. Thermal Anal. 1993, 39,1233

132. Yariv, S.; Lahav, N.; Lacher, M.; J. Thermal Anal. 1994, $42,13$.

133. Soma, Y.; Soma, M.; Furukawa, Y.; Harada, I.; Clays Clay Miner. 1987, 35, 53.

134. Yariv, S.; Nasser, A.; Michaelian, K. H.; Lapides, I.; Deutsch, Lahav, N.; Thermochim. Acta 1994, 234, 275.

135. Kodama, T.; Komarneni, S.; J. Mater. Chem. 1999, 9, 533. 